\title{
ESTRATÉGIAS DE DISPERSÃO, PRODUÇÃO DE FRUTOS E EXTRATIVISMO DA PALMEIRA Astrocaryum jauari MART. NOS IGAPÓS DO RIO NEGRO: IMPLICAÇÕES PARA A ICTIOFAUNA
}

\author{
M. T. F. Piedade ${ }^{1}$, P. Parolin ${ }^{2}$ y W. J. Junk ${ }^{2}$
}

\section{Resumo}

Astrocaryum jauari é a palmeira mais freqüente nos igapós do Rio Negro, na Amazônia brasileira, medindo em torno de $20 \mathrm{~m}$ de altura, encontrada raramente isolada e comumente formando touceiras com 4 a 6 indivíduos de tamanhos distintos, oriundos a partir de um único rizoma. A abscisão dos frutos ocorre durante o pico das cheias e inicio da descida das águas, sendo estes consumidos por peixes. A palmeira tem valor comercial sendo dela extraído palmito que, por cerca de 20 anos, até 1998, constituiu a base da produção industrial de palmito na Amazônia Central. Este estudo visou estabelecer a dinâmica de produção sazonal e biomassa de frutos de $A$. jauari em relação aos níveis de inundação de sua área de ocorrência e suas implicações em termos da dispersão e distribuição local da palmeira. Observações sistemáticas foram conduzidas na Estação Ecológica doArquipélago das Anavilhanas, perfazendo uma área total de levantamento de $5.000 \mathrm{~m}^{2}$. Determinou-se a densidade relativa de $A$. jauari em relação ao nível e duração da inundação anual. A produção de frutos foi acompanhada semanalmente em plantas individuai. Dados sobre peixes foram obtidos por meio de coletas, entrevistas e literatura. A. jauari ocorre das porções mais elevadas às mais baixas da planície alagável, inundadas de $270 \mathrm{a}$ 340 dias/ano. 125 indivíduos de $A$. jauari ocorreram nos $5.000 \mathrm{~m}^{2}$ amostrados, equivalendo a uma densidade de 250 ind/ha nas Anavilhanas. Apenas plantas adultas diretamente expostas à luz produziram frutos, cada estipe 4 cachos ao ano, com cerca de $90 \mathrm{~cm}$ de comprimento, e 106 frutos por cacho. Cada fruto pesou em média $17 \mathrm{~g}$, equivalendo à produção de $6,60 \mathrm{~kg} / \mathrm{palmeira} / \mathrm{ano}$ (peso fresco). 16 espécies de peixes exploram os frutos da palmeira, 10 possíveis dispersoras, dentre elas, 3 também predadoras. Pela ausência de estruturas para flutuação, quando na água os propágulos afundam e, se as condições forem hipóxicas, ocorrerá a morte do fruto e semente. Todas as sementes coletadas boiando estavam podres, descartando a hidrocoria como estratégia de dispersão e, apontando a ictiocoria como mecanismo para a colonização de novos habitats por $A$. jauari. O elevado conteúdo calórico de vitamina $\boldsymbol{A}$ pode ser atrativo para a busca desse recurso pelos peixes. Em porções alagadas por curtos períodos os frutos podem ficar acumulados próximos a planta adulta (barocoria), ou deslizar pelo relevo em virtude do seu peso, possivelmente com auxílio da ação da água (barocoria + deslizamento), aumentando a densidade local da planta. As espécies ocorrendo nas mesmas faixas de distribuição de $A$. jauari apresentam a hidrocória e ictiocoria como estratégias de dispersão predominantes, e grande tolerância a prolongados períodos de inundação. Na principal área de extrativismo da espécie no passado, no Município de Barcelos, a densidade de plantas também variou muito entre ilhas, porém, com densidades superiores àquelas encontradas neste estudo, variando de 250 a $2.000 \mathrm{ind} / \mathrm{ha}$. Nesse local, um único trabalhador pode derrubar e pré-limpar até 90 palmeiras na cheia (meses de maio, junho) e cerca de 25 na vazante (dezembro, janeiro). O rendimento por palmeira é de 0.5 a 1 lata de palmito processada. Supondo-se um número médio de 10 trabalhadores na área, de 900 a 250 palmeiras são exploradas ao dia, o que pode corresponder de 1 a 0.5 ha derrubados. Considerando as densidades da palmeira e sua produção de frutos calculada para as ilhas Anavilhanas, que foi de $165 \mathrm{~kg}$, o extrativismo sem plantio de reposição, implicaria na exclusão deste recurso para a ictiofauna daquele Arquipélago, com conseqüências negativas para as populações regionais e para o comércio íctico.

Palavras chave: Astrocaryum jauari, ictiocoria, extrativismo, igapós, produção de frutos, Amazônia, áreas alagáveis

\begin{abstract}
Astrocaryum jauari is the most frequent palmin the floodplains (igapó) of the Rio Negro, in Brazilian Amazonia. It grows to $20 \mathrm{~m}$ of height and is rarely found isolated. More commonly, 4-6 individuals of different sizes stand together which originate from the same rhizome. Fruit abscission occurs at highest water levels and dispersal occurs by fish. The palm has commercial
\end{abstract}

\footnotetext{
${ }^{1}$ INPA/Max Planck, C.P. 478, 69011 Manaus, Am, Brasil.

${ }^{2}$ Max-Planck-Institute for Limnology, Tropical Ecology, P.O. Box 165, 24302 Plön, Germany.
} 
value: until 1998, for 20 years 'palmito' was the basis of the industrial production in Central Amazonia. In the present study, seasonal dynamics and biomass of fruit production in $A$. jauari are described in relation to water level and annual flood duration. Systematic studies were performed at the Ecological Station of the Anavilhanas Archipelago, with an inventory area covering $5.000 \mathrm{~m}^{2}$. Fruit production was monitored at weekly intervals and biomass was determined. Data on the dispersing fishes was obtained by collection in the field and by interviews of local people. A. jauari occurs from the higher levels to the lowest in the flooding gradient, being waterlogged up to 270-340 days/year. 125 individuals of A. jauari occorred in the sampled $5.000 \mathrm{~m}^{2}$, which is equivalent to a density of $250 \mathrm{ind} / \mathrm{ha}$ in the Anavilhanas. Only adult trees which were directly exposed to light produced fruits. 16 species of fish feed on the fruits of $A$. jauari. Ten of these act as possible dispersers, 3 as predators. The high contents of vitamine $\boldsymbol{A}$ may be attractive for the fishes. Since the fruits do not have structures enhancing floatation, hydrochoric dispersal does not occur and the seeds succumb to the hypoxic conditions under water. In habitats with short periods of flooding, the fruits may be accumulated near the adult plant (barochory). Other trees occurring in the habitats colonized by $A$. jauari have hydrochoric and ichthyochoric dispersal syndromes and show high flooding tolerance. In the main area of extractivism of the past, in the Município Barcelos, the density of the species varied between the islands, and had densities between 250 and 2.000 ind/ha. Here, one worker could fell up to 90 palms at high water (May, June) and up to 25 in the low water period (december, january). For each tree, 0.5 to 1 bin of processed bin of "palmito" is produced. Assuming a mean number of ten workers in the area, 900 to 250 palms are exploited per day, corresponding to 1-0.5 ha destroyed forest. If we consid the densities of the palm and its productivity as calculated for the Anavilhanas islands (whcih was $165 \mathrm{~kg}$ ), extractivism without replacement of new palm trees implies a fast extinction and exclusion of this ressource for the ichthyofauna of the archipelago with all consequences for the local people.

Key words: Astrocaryum jauari, ichthyochory, extractivism, igapó, fruit production, Amazonia, floodplains.

\section{Introdução}

Dentre as poucas palmeiras de ocorrência nas áreas de inundação dos igapós do Rio Negro (Prance, 1980), a mais freqüente é Astrocaryum jauari (Figura $1 \mathrm{~A}$ y B), que mede em torno de 20 metros de altura, e apresenta o caule ornamentado por banda de acúleos, na extremidade do qual há uma pequena porém densa cabeça de folhagem. $O$ número de folhas contemporâneas é de 6 a 14 (Boer, 1965), tendo cada uma delas o comprimento médio de três metros. $A$. jauari pode ser encontrada raramente isolada e mais freqüentemente formando grandes grupamentos nas porções marginais dos rios e lagos, ou mais interiorizada na floresta alagada (Correa, 1969; Goulding, 1980). Quando agrupada, o número de indivíduos varia em geral de 4 a 6 , existindo na mesma touceira indivíduos de tamanhos distintos, sendo os clones formados a partir de um único rizoma (Boer, 1965).

Quando as águas no igapó atingem sua altura máxima e durante o início de sua descida, $A$. jauari elimina seus frutos. Estes são ovóides, medindo de 3 a $4 \mathrm{~cm}$, apresentando coloração entre verde e amarelada (Figura 1B). Assim como se verifica com outras plantas dessas comunidades, os frutos, ao caírem na água podem ser comidos por peixes, sendo então predados, caso sejam triturados, ou possivelmente transportados até locais adequados à sua germinação (Gottsberger, 1978; Goulding, 1980; Junk, 1980).

Comercialmente também pode ser extraído de $A$. jauari um palmito bastante apreciado por ser tenro e de sabor agradável, tendo sido a produção industrial de palmito da Amazônia Central até 1998, derivada exclusivamente dessa espécie. A partir desse ano, devido a facilidades de extração, transporte e constância na produção, o extrativismo passou a ser feito em plantações de Bactris gasipaes, popularmente chamada de pupunha (com. pess. Sr. Alírio Rocha, SHARP S/A).

Para os peixes da família Characidae, comercialmente importantes na região, em particular a pirapitinga (Colossoma bidens) e o tambaqui (Colossoma macropomum), os frutos de A. jauari constituem, durante a fase de alagação, respectivamente o primeiro e o segundo item alimentarpreferenciais (Goulding, 1980), contribuindo de maneira singular a alimentação regional, fortemente sustentada por proteína animal oriunda de peixe.

Este estudo objetivou determinar a dinâmica de produção e a biomassa de frutos de $A$. jauari em relação aos níveis de inundação de sua área de ocorrência, as implicações desses aspectos em temos da dispersão e distribuição local da palmeira, bem 


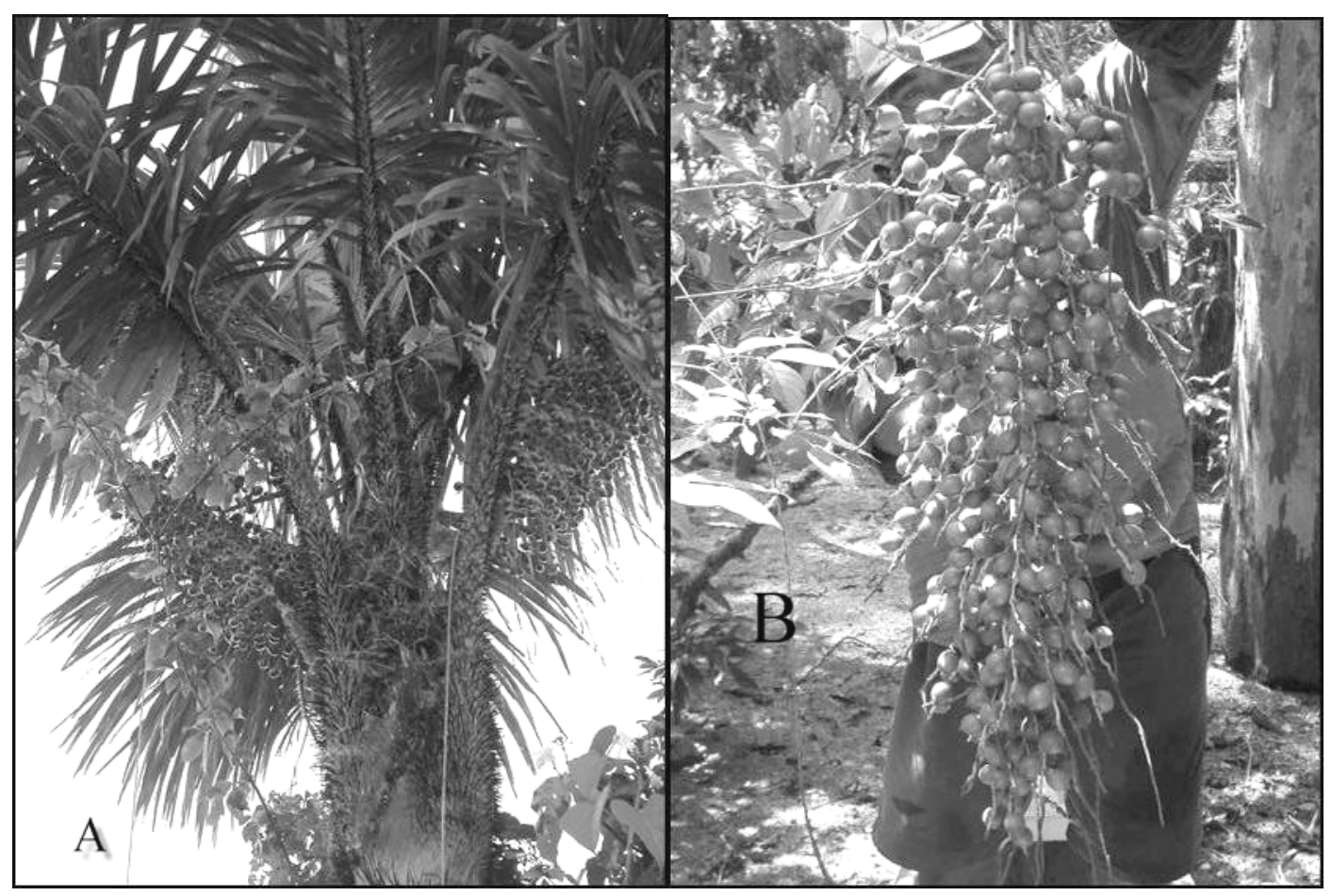

Figura 1. Astrocaryum jauari no ambiente natural. A Detalhe da árvore, B cacho de frutos (Fotos: F. Wittmann).

como o impacto de seu extrativismo nas cadeias alimentares desses ambientes.

\section{Área de estudo}

A base principal de dados de fenologia, produção de frutos e densidade da palmeira foi obtida de agosto de 1980 a novembro de 1982. Observações confirmatórias e informações sobre o extrativismo continuaram a ser acumuladas até 1998. A coleta de dados sobre fenologia e produção de frutos e densidade da espécie foi feita no Arquipélago de Anavilhanas, Rio Negro, Amazônia Central, na Estação Ecológica Flutuante do Instituto Brasileiro de Meio Ambiente (IBAMA), distante aproximadamente $100 \mathrm{~km}$ da cidade de Manaus (Figura 2).

Tomando como base dados climáticos de Manaus, segundo Ribeiro (1976), a região é caracterizada por um clima tropical quente e úmido (Afi de Köppen), com temperatura média inferior nunca abaixo de $18^{\circ} \mathrm{C}$ e o mês mais árido com uma umidade relativa superior a $60 \%$. A precipitação média anual para a região varia de 1000 a $2500 \mathrm{~mm}$, com a existência de uma estação seca no verão (Irmler, 1975). O nível do rio na área de estudo oscila ao longo do ano, com padrão e amplitude similares aqueles obtidos para o Rio Negro na altura da cidade de Manaus, apresentando um desnível médio de $10 \mathrm{~m}$ entre os picos das cheias e secas (Schmidt, 1973), que determina a presença de uma fase terrestre e uma fase aquática ao longo do ano, de grande importância biológica (Junk et al., 1989).

A vegetação da área apresenta em média três estratos e um sub-bosque pouco denso. As árvores mais altas apresentamentre 15 e $20 \mathrm{~m}$ de altura, e as emergentes ao redor de $25 \mathrm{~m}$. Durante as águas altas, dependendo do relevo das ilhas, a vegetação dessas áreas permanece em submersão total ou parcial, indicando a adaptação dos indivíduos a esse regime (Gessner, 1955; Fittkau, 1967; Irmler, 1973). Uma outra adaptação típica da vegetação desses ambientes é a eliminação dos frutos em sincronia com os níveis de águas altas, sendo por isso freqüentes a hidro e ictiocoria como mecanismos de dispersão (Gottsberger, 1978).

\section{Metodologia}

As observações foram centralizadas em dois sítios amostrais, próximos a sede flutuante do IBAMA (Figura 2). Para verificar as áreas de ocorrência e densidade relativa de $A$. jauari em relação às demais espécies presentes nos sítios amostrais, foram feitos dois transectos, um no sítio amostral 2 e outro no sítio amostral 3 (Figura 3). Os transectos estenderam-se do litoral da ilha até a porção do barranco, percorrendo toda a superfície transversal em cada um deles. Assim, foi realizado no sítio $2 \mathrm{um}$ transecto de $20 \times 115 \mathrm{~m}$ $\left(2300 \mathrm{~m}^{2}\right)$, e no sítio $3 \mathrm{um}$ transecto de $20 \times 135 \mathrm{~m}$ $\left(2.700 \mathrm{~m}^{2}\right)$. Essas faixas foram divididas em quadrados de $20 \mathrm{~m}$, em cada um dos quais procedeuse ao levantamento de todos os indivíduos com DAP superior a $10 \mathrm{~cm}$, sendo feito um mapeamento para verificar a posição relativa de $A$. jauari e demais espécies presentes. Com os valores encontrados determinou-se a densidade relativa (DR) de cada espécie presente por faixa de $20 \mathrm{~m}$, segundo Cottam \& 
Curtis (1956), onde $\mathrm{DR}=\mathrm{n} \times 100 / \mathrm{n}_{\mathrm{t}}(\mathrm{n}=$ número de indivíduos da espécie; $\mathrm{n}_{\mathrm{t}}=$ número de indivíduos de todas as espécies presente na área amostrada).

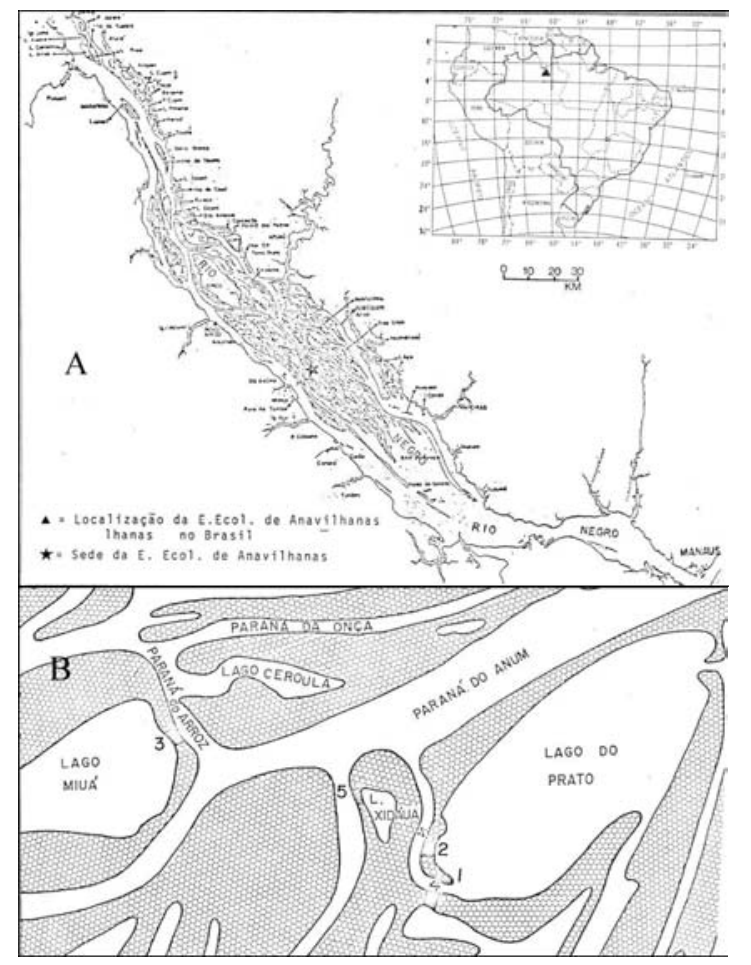

Figura 2. A Arquipélago de Anavilhanas, Rio Negro, Amazônia Central, distante aproximadamente $100 \mathrm{~km}$ da cidade de Manaus; B sítios amostrais próximos a Estação Ecológica Flutuante do Instituto Brasileiro de Meio Ambiente (IBAMA).

Destacou-se a presença de $A$. jauari em relação ao nível de inundação. O cálculo do número de dias de alagação ao qual as comunidades vegetais estão submetidas por faixa de relevo, foi realizado tomando como base a curva do valor médio de dez anos de dias de inundação confeccionada por Junk et al. (1983), com as cotas do Rio Negro, na altura de Manaus, fornecidas pela PORTOBRÁS. As demais espécies presentes foram agrupadas por família, e ordenadas de forma decrescente, conforme o número de espécies componentes.

Para a obtenção de dados sobre a dinâmica de produção de frutos e aspectos de dispersão, foram realizadas observações gerais em todo o Arquipélago, bem como em todos os indivíduos dos 3 sítios amostrais (Figura 3), e, particularmente em 3 indivíduos marcados nos sítios 1,2 e 3, em um total de 9 indivíduos acompanhados. Os registros foram feitos pelo menos uma vez ao mês e pelo menos uma vez por semana, durante a fase de queda de frutos. Com o auxilio de rapixé, procedeu-sea coleta de sementes de $A$. jauari flutuando na água, num total superior a 250, além de coletas casuais. Todas as sementes foram quebradas para verificar as condições da amêndoa.

Além das observações gerais, foram coletados dez cachos de frutos de palmeiras diferentes, sempre marginalmente dispostas. Os cachos coletados foram medidos, tendo sido contado o número total de frutos presentes. A seguir, de cada um dos cachos retirou-se uma sub-amostra de 20 frutos, dos quais foi tomado o peso fresco utilizando-se uma pesola. Para verificar as formas de exploração dos frutos pela fauna do igapó, observou-se mais de 3000 frutos no solo (ataque pósdispersão) e mais de 1000 frutos em cachos (ataque pré-dispersão). Como parte de outros estudos em andamento, a coleta de peixes realizada sistematicamente durante as cheias na área da Estação Ecológica, forneceu-se dados a respeito da ingestão de frutos por peixes. A esses dados foram acrescentados os da bibliografia e de entrevistas com moradores da região. Dados sobre o extrativismo da palmeira até de 1998, foram fornecidos por técnicos da Fábrica de Palmito Jauari, SHARP do Brasil S.A., Manaus/AM.

\section{Resultados}

A distribuição de indivíduos de A. jauari, variou bastante entre os dois sítios amostrais. No sítio amostral 2, apenas 4 indivíduos adultos de $A$. jauari foram encontrados. Estes mediam acima de $14 \mathrm{~m}$ de altura e frutificaram durante o período de estudo. Desse total, 3 distribuíram-se nos 40 primeiros metros da porção litoral em direção a parte mais elevada da ilha. O quarto indivíduo foi encontrado na porção do barranco, em uma altura equivalente a faixa de ocorrência da porção litoral. O número de plântulas apresentou-semaior ao redor dos adultos, em particular nas porções litorais, as menos íngremes do relevo. As faixas de ocorrência de A. jauari, foram as que apresentaram maior diversidade de espécies nessa estação, tendo sido encontradas 29 espécies associadas (Tabela 1 y 2). Contudo, ao longo da ilha, observou-se a substituição de dominantes conforme o tempo de alagação e a altura do relevo. Neste sítio amostral, observou-seque a palmeira se distribuiu em uma faixa que permanece inundada, pelo menos na base do caule e sistema radicular, de 270 a 340 dias, o mesmo ocorrendo com as espécies a ela associadas.

O sítio amostral 3 apresentou uma densidade muito mais elevada de indivíduos de A. jauari. O número total de indivíduos com mais de $14 \mathrm{~m}$ de altura foi de 121. Neste sítio, onde a porção litoral apresentou-se muito plana, os adultos de $A$. jauari situaram-se apenas a partir dos primeiros $60 \mathrm{~m}$ em relação ao litoral da ilha. Na porção mais isolada do relevo predominaram indivíduos clonais, enquanto que nos $20 \mathrm{~m}$ anteriores e no barranco, predominaram adultos isolados com plântulas e jovens ao seu redor. Considerando toda a faixa de ocorrência da palmeira 
em este sítio amostral, encontrou-se 27 espécies associadas a ela, distribuídas na porção litoral e do barranco. Da mesma forma que no sítio amostral 2,observou-se a substituição de espécies dominantes, conforme o tempo de inundação. Nesta área A. jauari ocorreu numa faixa de relevo submetida de 30 a 200 dias de inundação ao ano, dependendo da posição mais ou menos elevada da ilha.

Considerando as duas áreas de estudo, num total de $5.000 \mathrm{~m}^{2}$ amostrados, foram encontrados 125 indivíduos, o que fornece uma densidade para $A$. jauarinas Ilhas Anavilhanas, de 250 indivíduos por hectare.

A freqüência de produção de flores e frutos de $A$. jauari é anual. A grande maioria das palmeiras adultas inicia a produção de flores quando as águas do rio estão descendo, especialmente durante os meses de agosto e setembro. A fase de floração é rápida, sendo logo substituída pela presença de pequenos frutos que vão desenvolver-se lentamente, amadurecendo apenas quando as águas do rio sobem novamente, sofrendoabcisão principalmente quando elas atingem seus níveis mais altos, nos meses de maio, junho e julho. Tanto as fases de floração quanto os primeiros estágios de frutos jovens transcorrem durante os meses nos quais as águas estão descendo e a precipitação ainda não é muito intensa. $\mathrm{O}$ tempo gasto para a produção de frutos é em média de 9 meses. Verificou-se também que as palmeiras, ainda que possivelmente velhas pela presença de caule glabro e repleto de liquens (Boer, 1965), não produziram cachos caso estivessem abaixo do dossel ou em qualquer posição na qual não recebessem luz. Em áreas isoladas, mesmo aquelas pequenas $\mathrm{e}$ aparentemente jovens, já frutificavam.

Cada palmeira individual de $A$. jauari, produz anualmente ao redor de 4 cachos, que medem cerca de $90 \mathrm{~cm}$ de comprimento. O número médio de frutos por cacho é de 106 e o peso médio por fruto é de $17 \mathrm{~g}$. Devido a esse peso e a ausência de estruturas bem desenvolvidas para a flutuação, como é comum em outras palmeiras como Cocos nucifera, todos os frutos afundam ao cair na água. Por sua vez, todas as sementes coletadas boiando apresentavam a amêndoa podre.

Como conseqüência das condições topográficas, após a queda, os frutos podem ficar acumulados próximo a planta adulta (Barocoria), ou deslizar pelo relevo em virtude do seu peso, possivelmente com auxílio da ação da água (Barocoria + Deslizamento). Os frutos encontrados nessa circunstâncias são em menor número e isolados em pontos esparsos do relevo insular.

Um total de 16 espécies de peixes exploram os frutos da palmeira, pertencentes a cinco famílias, das quais Characidae é a mais representada. Desse total de espécies, 10 foram categorizadas como possíveis dispersoras (Ictiocoria), embora 3 delas (Colossoma macropomum, Colossoma bidens e Prachtocephalus hemiliopterus), possam também atuar como predadoras, já que além de engolirem frutos inteiros, podem fragmentá-los com suas possantes mandíbulas.

Durante este estudo, não observamos indícios de utilização dos frutos de A. jauari por nenhum outro vertebrado.

Quanto a exploração econômica da espécie, as informações fornecidas pela Fábrica de Palmito Jauari - SHARP do Brasil S.A., dão conta que na região do Município de Barcelos, onde o extrativismo ocorre, a densidade de plantas também varia muito entre ilhas daquela localidade, porém, as densidades são muito superiores àquelas encontradas no Arquipélago de Anavilhanas (250 indivíduos por hectare), sendo da ordem de 250 até 2.000 indivíduos por hectare. Nesse local, um único trabalhador pode derrubar e prélimpar até 90 palmeiras na cheia (meses de maio, junho) e cerca de 25 na vazante (dezembro, janeiro). Supondo-se um número médio de 10 trabalhadores na área, de 900 a 250 palmeiras são exploradas ao dia, o que pode corresponder de 1a 0.5 ha. O rendimento por palmeira é de 0.5 a 1 lata de palmito processada. Atualmente, desde outubro de 1998, devido a dificuldades em se manter constância na produção a produção de palmito da SHARP S.A., foi totalmente substituída por pupunha (Bactris gasipaes), plantadas em 45 ha manejados em um total de 225.000 palmeiras.

\section{Discussão}

Nos sítios amostrais verificou-se uma nítida substituição de comunidades ao longo da topossequência das ilhas, como resposta as variações do número de dias de alagação, e em função da compactação do solo, que aumenta em direção as 
Diciembre 2003

TABELA 1: LISTAGEM TOTAL DE ESPÉCIES ENCONTRADAS EM $2300 \mathrm{M}^{2}$ NA ESTAÇÃO DE ESTUDO 2 - LAGO DO PRATO (DAP $\geq 10 \mathrm{~cm}$ ).

\begin{tabular}{|c|c|c|c|}
\hline FAMILIA & 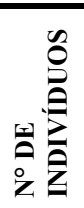 & 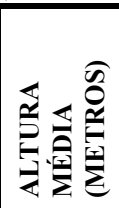 & ESPÉCIES \\
\hline APOCYNACEAE & 5 & 11,0 & Malouetia furfuracea Benth. Ex Muell. Arg. \\
\hline \multirow{2}{*}{ ANNONACEAE } & 2 & 11,5 & Guatteria sp. \\
\hline & 5 & 13,8 & Unonopsis sp. \\
\hline BURSERACEAE & 4 & 12,3 & Protium sp. \\
\hline \multirow[t]{4}{*}{ CHRYSOBALANACEAE } & 1 & 14,0 & Couepia paraensis (Mart. \& Zucc.) \\
\hline & 1 & 9,0 & Hirtellasp. \\
\hline & 1 & 9,0 & Hirtella racemosa Lam. Var. racemosa \\
\hline & 5 & 14,5 & Licania sp. \\
\hline COMBRETACEAE & 1 & 13,0 & Buchenawia oxycarpa Eichl. \\
\hline \multirow[t]{2}{*}{ EUPHOBIACEAE } & 1 & 14,0 & Amanoa oblongifolia M. Arg. \\
\hline & 1 & 12,0 & Mabea nitida Spruce ex Benth. \\
\hline \multirow[t]{4}{*}{ GUTTIFERAE } & 3 & 10,4 & Tovomita sp. \\
\hline & 1 & 13,0 & Rheedia acuminata Pl. et Triana \\
\hline & 1 & 10,0 & Caraipa sp. \\
\hline & 2 & 11,5 & Vismia sp.. \\
\hline LAURACEAE & 1 & 13,0 & Ocotea cf. fasciculata (Nees) Mez. \\
\hline \multirow[t]{2}{*}{ LECYTHIDACEAE } & 2 & 14,0 & Eschweilera coriacea(DC.) Mart. Ex Berg \\
\hline & 2 & 11,0 & Eschweilera sp. \\
\hline \multirow{13}{*}{ LEGUMINOSAE } & 2 & 17,0 & Swartzia laevicarpa Amsh. \\
\hline & 6 & 15,8 & Tachigalia myrmecophilla (Ducke) Ducke \\
\hline & 1 & 13,0 & Sclerolobium aff. Odoratissimum. Sorece wx Benth. \\
\hline & 5 & 19,2 & $\begin{array}{l}\text { Peltogyne venosa (Vahl.) Benth. Subsp densiflora (Spruce ex Benth.) M. F. Silva. } \\
\text { Swartzia polyphylla DC. }\end{array}$ \\
\hline & & & Crudia sp. \\
\hline & 1 & 17,0 & Aldina latifolia Spruce ex Benth. \\
\hline & 6 & 16,2 & Elizabetha speciosa Ducke \\
\hline & 2 & 14,0 & Inga sp. \\
\hline & 4 & 15,0 & Campsiandra comosa Benth. \\
\hline & 3 & 14,7 & Tachigalia paniculata Aubl. \\
\hline & 2 & 21,5 & Tachigalia sp. \\
\hline & 2 & 16,0 & \\
\hline & 1 & 13,0 & \\
\hline MELIACEAE & 1 & 14,0 & Trichilia sp. \\
\hline MYRCIANACEAE & 1 & 16,0 & Cybianthus spicatus (H.B.K.) Agost. \\
\hline MYRISTICACEAE & 1 & 14,0 & Virola elongata (Bth.) Warb. \\
\hline MORACEAE & 1 & 11,0 & Cecropia sp. \\
\hline ARECACEAE & 4 & 16,5 & Astrocaryum jauari Mart. \\
\hline RUBIACEAE & 2 & 11,0 & Duroia velutina Hook. Et Schum \\
\hline SAPINDACEAE & 1 & 14,0 & Natayba sp. \\
\hline SAPOTACEAE & 1 & 14,0 & Pouteria elegans (A. D. C. ) Aubr. \\
\hline TILIACEAE & 9 & 13,4 & Nollia speciosa Mart. Et Zucc. \\
\hline VIOLACEAE & 1 & 8,0 & Rinorea sp. \\
\hline & 1 & 9,0 & Payparola sp. \\
\hline
\end{tabular}

porções mais elevadas do relevo insular. Tais amplitude de distribuição em relação a alagação, como variações de comunidades ao longo de gradientes de inundação já foram descritas por vários autores para o Rio Negro (Takeuchi, 1962; Revilla, 1981; Worbes, 1983; Wittmann \& Junk, 2003). Já em 1906, Huber separou a vegetação do Rio Purús, desde as áreas alagadas até as porções mais elevadas, com base nas espécies dominantes. Louis (1947), discute o mesmo tipo de estratificação em comunidades das ilhas Yangambi, no Congo. No caso deste estudo, foram encontradas espécies com maior densidade relativa em diferentes faixas do relevo, porém, considerando as duas áreas amostradas, as espécies diferiram. As espécies dominantes encontradas no sítio amostral 3 foram em menor número, e demonstraram uma maior é o caso típico de Nectandra amazonun, que permanece em faixas do relevo submetidas de 180 a 340 dias de alagação. Considerando ambas as áreas, Tovomita sp e Coccoloba pichuna foram as espécies que ocorreram exclusivamente nos pontos do relevo submetidos aum maior número de dias de inundação durante o ano (cerca de 340 dias).

Quanto a $A$. jauari, a distribuição e densidade variaram muito entre as duas ilhas. No sítio amostral 2, a palmeira apresentou-se em áreas do relevo que permanecem inundadas de 270 a 340 dias ao longo do ano, enquanto que no sítio amostral 3, ela ocorreu em áreas inundadas de 30 a 200 dias ao longo do ano. Essa grande amplitude de distribuição já havia sido 
apontada por Huber (1906), para populações de $A$. jauari do rio Purus. Uma vez que a espécie apresenta grande tolerância a inundação, a diferença no número de indivíduos e na faixa de distribuição entre as duas ilhas amostradas, possivelmente deve-se a diferenças no tempo de colonização entre elas.

TABELA 2: LISTAGEM TOTAL DE ESPÉCIES ECONTRADAS EM $2700 \mathrm{M}^{2}$ NA ESTAÇÃO DE ESTUDO 3 LAGO MIUÁ (DAP $\geq 10 \mathrm{~cm}$ ).

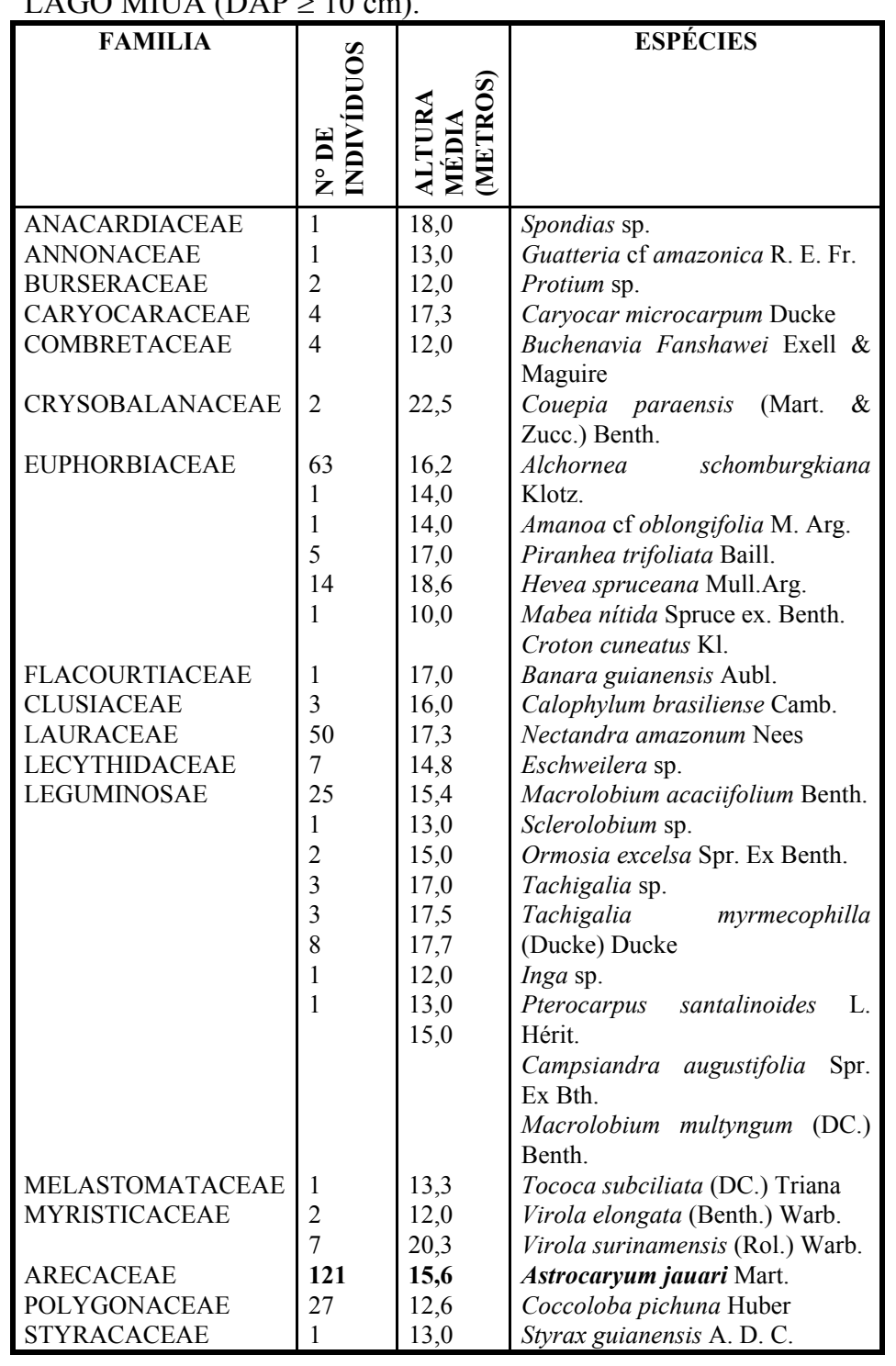

palmeira produz em torno de $6,60 \mathrm{~kg}$ de frutos ao ano (peso fresco). Entretanto, a pressão para uma sincronia na produção de frutos nas fases de águas mais altas pode ser mais importante, por ser compatível com os mecanismos de dispersão da espécie. Também o alto valor energético e nutritivo dos frutos pode explicar a grande procura desse recurso pelos peixes. O elevado conteúdo calórico de vitamina $\mathrm{A}$ pode ser especialmente vantajoso se consumido em grande quantidades durante as cheias, para estocar reservas na forma de gordura para as fases de deslocamento, de desova ou de águas baixas, quando os recursos alimentares são escassos. Confirmando a preferencia dos peixes por esse recurso, Goulding (1980) encontrou em apenas um indivíduo de Colossoma macropomum 52 frutos inteiros, e em um indivíduo de Colossoma bidens $1 \mathrm{~kg}$ de frutos de $A$. jauari.

A constatação de que todos os frutos encontrados boiando estavam podres, indica que a hidrocoria não se verifica com A. jauari. Ao cairem na água, os pesados frutos vão para o fundo, podendo eventualmente ser transportados por correstes submersas, mas não por flutuação. Nesses locais, eles poderão germinar, em função da profundidade e do nível da água durante a fase terrestre quando o local da queda é muito profundo, condições hipóxicas levam ao apodrecimento do fruto e semente que passa então a boiar. Corner (1966) salienta ser freqüente a "aparente" dispersão por flutuação em alguns gêneros de palmeiras, citando inclusive Astrocaryum entre eles.

Assim, o aumento local na densidade de espécie é decorrente da barocoria e da barocoria + deslizamento, que associadas à propagação vegetativa propiciam o aparecimento de grandes massas de indivíduos da espécie nas ilhas Anavilhanas e densidades ainda maiores na região de seu extrativismo em Barcelos.

A. jauari sincroniza sua floração com o período no qual a precipitação é pouco intensa, e, quando os índices pluviométricos maiores são obtidos, os frutos já estão em processo de crescimento, um processo longo que dura em torno nove meses, até o pico das águas altas, período mais intenso de abcisão dos mesmos. Possivelmente esse longo período é necessário em virtude do alto conteúdo energético e vitamínico dos frutos exigir um longo processo de bombeamento dos escassos nutrientes desses solos pobres (Sioli \& Klinge, 1962; Furch, 1997). Cada
Uma vez que a hidrocoria não se verifica, a distribuição da espécie a longas distancias para a colonização de novos habitats, é decorrente da ictiocoria. Segundo Van der Pijl $(1969,1972)$, a ictiocoria é um mecanismo de dispersão primitivo que em áreas inundáveis da Amazônia e Borneo se mantêm nos grupos de plantas mais antigos. O ruido da queda dos frutos na água, atraindo os peixes, contribui para o sucesso desse mecanismo. 


\section{Bibliografia}

Ab'Saber A.N. 1967. Problemas geomorfológicos da Amazônia brasileira. Atas do Simpósio sobre a Biota Amazônica 1 (Geociências).: 35-67.

Adis J. 1977. Programa mínimo para análises de ecossistemas: artrópodos terrestres em florestas inundáveis da Amazônia Central. Acta Amazônica. 7(2): 223-229.

A.O.A.C. 1975. Handbook of chemical analysis. $10^{\text {a }}$ ed. Washington, Association of Official Analytical Chemist.

Boer J.E.W. 1965. Palmae. In: Ianjouw, J. (ed.) Flora of Suriname . vol. 5. pate 1. Leiden E. J. Brill. : 125-141.

Braga P.I.S. 1979. Subdivisão fitogeográfica, tipos de vegetação conservação e inventário florístico da floresta amazônica. Acta Amazônica. 9(4): 53-80 .

Corner E.J.H. 1966. The natural history of palms. London, Weidenfeld and Nicholson .

Corréa M.P. 1969. Dicionário de plantas üteis do Brasil. vol. 4. IBDF. Rio de Janeiro.

Cottam G. \& Curtis J.T. 1956. The use of distance measures in phytosociological sampling. Ecology. 7(3): 451-460.

Coatinho L.M. \& Lamberti A. 1971. Respiração edáfica e produtividade primária numa comunidade amazônica de terra firme. Ci. e Cult. 23(3): 411-419.

Cronquist A. 1974. The evolution and classification of flowering plants Boston, Houghton Mifflin Co.

Ducke A. \& Black G.A. 1953. Notas sobre a fitogeografia da Amazônia brasileira. Bol.Têc.Inst. Agron. Norte. 29: 1-62.

Duvigneaud F. 1946. La variabilité des associations vêgitales. Bull. Soc. Dot. Belg. 78: 107-134.

Erwin T.L. \& Adis J. 1982. Amazonian inundation forests their role as short-term refuges and generators of species richness and taxon pulsesIn: Prance , G.T. ed.-Biologicaldiversification in the tropics . New York ,Columbia.University Press. : 358-371.

Fittkau E.J. 1964. Remarks on limnology of Central Amazon rain-forest stream. Verh.Internat. Verein.Limnol. 15: 1092-1096.

Fittkau E.J. 1967. On the ecology of amazonian rainforest streams. Atas do Simpósio sobre a Biota Amazônica 3(Limnologia).: 97-108.

Fittkau E.J. 1971. Esboço de uma divisão ecológica da região amazônica . In: Idrobo, J.M. (ed.). II Simpósio y Foro de Biologia Tropical Amazônica. Bogotá, Editorial PAX.: 365-372.

Fittkau E.J., Junk W.J., Klinge H. \& Sioli H. 1975a. Substrate and vegetation in the Amazon region.In: Dierschke, Ii. (ed.). Vegetation und substrat. Vaduz, J. Cramer.: 73-90.

Fittkau E.J., Irmler U., Junk W.J., Reiss F. \& Schmidt G.W. 1975b. Productivity, biomass, and population dynamics in amazonian water bodies . In: Golley, F. B.\& Medina, E. (eds.). Tropical ecological systems: trends in terrestrial and aquatic research. New York, Springer Verlag.: 289-311.

Fleming T.H. \& Iieithaus E.R. 1981. Frugivorous bats, seed shadows and the struture of tropical forests. Biotropica. 13(2): 45-53.

Furch K. 1997. Chemistry of várzea and igapó soils and nutrient inventory in their floodplain forests. In: The Central Amazon floodplain: Ecology of a pulsing system. Junk W.J. (ed.). Ecological Studies 126, Springer Verlag, Heidelberg. : 47-68.

Gessner F. 1955. Hydrobotanik. Band I: Energiehaushalt. Berlin, VEB Deutscher Verlag der Wissenschaften.

Gibbs R.J. 1967. The geochemistry of the Amazon river system. Part I. The factors that control the salinity and the composition and concentration of the suspended solids. Geol. Soc. Am. Bull. 78: 1203-1232.

Gottsberger G. 1978. Seed dispersal by fish in the inundated regions of Humaitá, Amazônia.Biotropica. 10 (3): 170-183.

Goulding M. 1979. Ecologia da pesca no rio Madeira. Manaus, INPA.

Goulding M. 1980. The fishes and the forest. Los Angeles, Univ. of California Press.

Harper J.L. 1977. Population biology of plants. New York, Academic Press.

Huber J. 1906. La végétation de la vallée du rio Purus ( Amazone ). Bulletin de L'Herbier Boissier, 2me série. 4(4): 249-276.

Illies J. 1967. Versuch einer allgemeinen biozönotischen Gliederung der Fliessgewässer Int. Revue Hydrobiol. 46(2): 205-213.

Irio G. \& Adis J. 1979. Evolução de florestas amazônicas inundadas, de igapó - um exemplo do rio Tarumã-mirim. Acta Amazonica. 9(2): 299-303.

Irmler U. 1973. Population-dynamic and physiological adaptation of Pentacomia egregia CHAUD. (Col. Cicindelidae) to the amazonian inundation forest. Amazoniana. 4(2): 219-227.

Irmler U. 1975. Ecological studies of the aquatic soil invertebrates in three inundation forests of Central Amazônia. Amazoniana. 3(3): 337-409.

Irmler U. 1977. Inundation-forest tipes in the vicinity of Manaus. Biogeographica. 8: 17-29.

Irmler U. 1978. Matas de inundação da Amazônia Central em comparação entre águas brancas e pretas. Ci. e Cult. 30(7): 813-821.

Janzen D.H. 1967. Synchronization of sexual reprodution of trees within the dry season in Central América. Evolution. 21(3): 620-637.

Janzen D.H. 1969. Seed-eaters versus seed size, number, toxicity and dispersal. Evolution. 23(1): $1-27$. 
Janzen D.H. 1970. Herbivores and the number of tree species in tropical forests. Am. Nat. 104 (940): 501-528.

Janzen D.H. 1971. Seed predation by animals . A. Rev. Ecol. Syst. 2: 465-492.

Janzen D.H. 1972. Association of a rain-forest palm and seed-eating beetles in Puerto Rico. Ecology. 53(2): 258-261.

Janzen D.H. 1974. Tropical blackwater rivers, animals, and mast fruiting by the Dipterocarpaceae. Biotropica. 6 (2): 69-103.

Junk W.J. 1970. Investigations on the ecology and production-biology of the "floating meadows" (Paspalo Echinocloetum) on the Middle Amazon . I . The floating vegetation and its ecology . Amazoniana. 2(4): 449-495.

Junk W.J. 1980 . Áreas inundáveis - Um desafio para limnologia. Acta Amazonica. 10: 775-795.

Junk W.J. 1983. Ecology of swamps of the Middle Amazon. In: Goodall, D.W. ed. - Ecosystems of the world . Amsterdam, Elsevier Scientific Publishing Co. : 269-294.

Junk W.J. \& Furch K. 1980. Química da água e macrófitas aquáticas de rios e igarapés da bacia amazônica e nas áreas adjacentes . Parte I. Trecho Cuiabá - Porto Velho - Manaus. Acta Amazonica. 10(3): 611-633.

Junk W.J., Klinge H., Revilla J.C., Worbes M. \& Furch B. 1983. A floresta inundável . In : INPA Relatório anual.: 194-198.

Junk W.J., Bayley P.B. \& Sparks R.E. 1989. The flood pulse concept in river-floodplain systems. In: Proceedings of the International Large River Symposium. Dodge D.P. (ed.). Can. Publ. Fish. Aquat. Sci. 106: 110-127.

Keel S.H.K. \& Prance G.T. 1979. Studies of the vegetation of a white and black-water igapó ( Rio Negro Brazil ) . Acta Amazônica. 9 (4): 645-655.

Klinge H. 1965. Podzol soils in the Amazon basin. J. Soil Sci. 16(1): 95-103.

Leenheer J.A. \& Santos H. 1980. Considerações sobre os processos de sedimentação na água preta ácida do rio Negro (Amazônia Central). Acta Amazônica. 10(2): 343-355.

Leite A.M.C. \& Rankin J.M. 1981. Ecologia de sementes de Pithecolobium racemosumDucke. Acta Amazônica. 11 (2): 309-318 .

Louis L. 1947. L'origine et la vegétation des iles du fleuve de la région de Yangambi . C.R. Sem. Ar. Yangambi. 12: 924-933.

Luizão F.J. 1982. Produção e decomposição da liteira em floresta de terra-firme da Amazónia Central . Aspectos químicos e biológicos da lixiviação e remoção dos nutientes da liteira. Tese de Mestrado Mánaus , INPA/ FUA.

Machado-Allison A.J. 1982. Studies on the systematics of the subfamily Serrasalminae (
Pisces-Characidae). Ph. D. Thesis. Washington, The George Washington University.

Martius K.F.P. von. 1823-1850. Historia naturalis palmarum. vol 1-3. Lipsiae, T.O. Weigel.

Moore H.E.Jr. 1973a . Palms in the tropical forest ecosystems of Africa and South Amarica. In: Meggers, D. J. ; Ayensu , E. S. ; Duckworth , W. D. eds. - Tropical forest ecosystems in Africa and South America : a comparative review '. Washington, Smithsonian Institution Press. : 6388.

Moore H.E. Jr. 1973b. The major groups of palms and their distribution. Ithaca, Cornell University.

Odum E.P. 1971. Ecologia. México, Nueva Editorial Interamericana.

Pianka E.R. 1970. On r-and K- selection . Am. Nat. 104: 592-597.

Pearson D. 1975. Laboratory techniques in food analysis. London, Butterworth \& Co.

Pires J.M. 1974. Tipos de vegetação da Amazônia. Brasil Florestal. 5(17): 49-58.

Prance G.T. 1978. The origin and evolution of the Amazon flora. Interciência. 3(4): 207-222.

Prance G.T. 1980. A terminologia dos tipos de florestas amazônicas sujeitas à inundação. Acta Amazônica. 10(3): 495-504.

RADAMBRASIL. 1978. Levantamento de recursos naturais. Folha SA. 20. Manaus. Rio de Janeiro, Departamento Nacional de Produção Mineral.

Rankin J.M. 1978. The influence of predation on tree species abundance in two adjacent rain forest communities in Trinidad, West Indies. Ph.D. Thesis. Ann Arbor, University of Michigan.

Revilla J.D.C. 1981. Aspectos florísticos e fitossociológicos da floresta inundável (igapó). Praia Grande, rio Negro, Amazonas, Brasil. Dissertação de Mestrado. Manaus, INPA/FUA.

Ribeiro M.C.L.B. 1983. As migrações dos jaraquis (Pisces, Prochilodontidae) no rio Negro, Amazonas, Brasil. Dissertação de Mestrado. Manaus, INPA/FUA.

Ribeiro M.N.G. 1976. Aspectos climatológicos de Manaus. Acta Amazonica. 6(2): 229-233.

Rodrigues W.A. 1961. Estudo preliminar de mata de várzea alta de uma ilha do baixo rio Negro de solo argiloso e úmido. Publicação do INPA - série Botânica. 10.

Sarukhán J. 1974. Studies on plant demography: Ranunculushepens L., R. bulbosus L. e R. acris L. II. Reproductive strategies and seed population dynamics. J. Ecol. 62(1): 151-177.

Schmidt G.W. 1973. Primary production of phytoplancton in the three types of amazonian waters. II. The limnology of a tropical flood-plain lake in Central Amazonia (Lago do Castanho). Amazoniana. 4(2): 139-203. 
Schmidt G.W. 1976. Primary production of phytoplancton in the three types of amazonian waters. IV. On the primary productivity of phytoplancton in a bay of the lower rio Negro (Amazonas, Brazil). Amazoniana. 5(4): 517-528.

SEMA. 1982. Programa de estações ecológicas. Brasília, Ministério do Interior.

Sioli H. 1951. Alguns resultados e problemas da limnologia amazônica. Bolm tëc. Inst. agron. N. 24: 2-44.

Sioli H. 1964. General features of the limnology of Amazonia. Verh. Internat. Verein. Limnol. W5(2): 1053-1058.

Sioli H. 1965. A limnologia e sua importância em pesquisas na Amazônia. Amazoniana. 1(1): 11-35.

Sioli H. 1968. Hydrochemistry and geology in the brazilian Amazon region. Amazoniana. 1(3): $267-$ 277.

Sioli H. 1973. Principais biótopos de produção primária nas águas da Amazônia. B. geogr. (R. Janeiro). 32(236): 131-142.

Sioli H. 1975. Tropical river: the Amazon. In: Whitton, B. A. ed. - River ecology. Berkeley, Univ. of California Press. : 461-488.

Sioli H. 1976. A limnologia na região amazônica brasileira. Anais do I Encontro Nacional sobre Limnologia, Piscicultura e Pesca Continental. Belo Horizonte. : 153-169.

Sioli H. \& Klinge H. 1962. Solos, tipos de vegetação e águas na Amazônia Brasileira. B. Mus. Paraen. Emilio Goeldi, nova sér., Avulsa. 1: 27-41.
Takeuchi M. 1962. The structure of the amazonian vegetation. VI. Igapó. Journ. Fac. Sci. Univ. Tokyo, III. 8(7): 297-304.

Yeaton R.I. 1979. Intraespecific competition in a population of the stilt palm, Socratea duríssima (Oerst.) Wendl. on Barro Colorado island, Panamâ. Biotropica. 11(2): 115-158.

van der Pijl L. 1969. Evolutionary action of tropical animals on the reproduction of plants. Biol. J. Linn. Soc. 1: 85-96.

van der Pijl L. 1972. Principes of dispersal plants. Berlin, Springer-Verlag. 161 p. in higher planta. Berlin, Springer-Verlag.

Whitmore T.C. 1975. Tropical rain forest of the far east. Oxford, Clarendon Press.

Wilson D.E. \& Janzen D.H. 1972. Predation of Scheelea palm by bruchid beetles: seed density and distance from the parent palm. Ecology. 53: 954-959.

Wittmann F. \& Junk W.J. 2003. Sapling communities in Amazonian white-water forests. Journal of Biogeography. 30(10): 1533-1544.

Worbes M. 1983. Vegetationskundliche Untersuchungen zweier Überschwemmungswälder in Zentralamazonien - vorläufige Ergebnisse. Amazoniana. 8(1): 47-65.

Worbes M. 1984. Periodische Zuwachszonen an Bäumen zentralamazonischer Überschwemmungswälder. Naturwissenschaften. $71: 157$. 